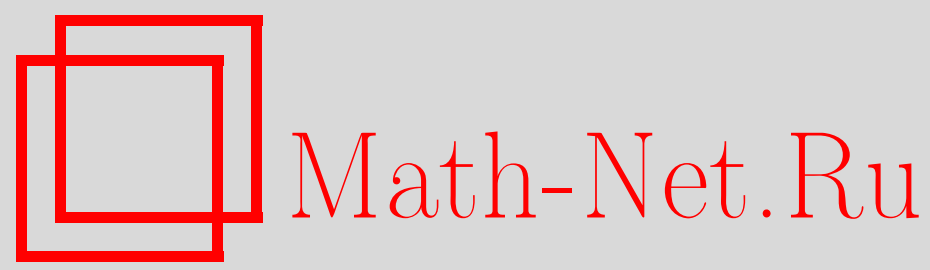

Н. В. Литвинов, Об областях определения $t$-функций, $M a$ тем. заметки, 2003, том 73, выпуск 4, 573-576

DOI: https://doi.org/10.4213/mzm205

Использование Общероссийского математического портала Math-Net.Ru подразумевает, что вы прочитали и согласны с пользовательским соглашением http://www . mathnet.ru/rus/agreement

Параметры загрузки:

IP: 52.205 .19 .152

26 апреля 2023 г., 09:02:37

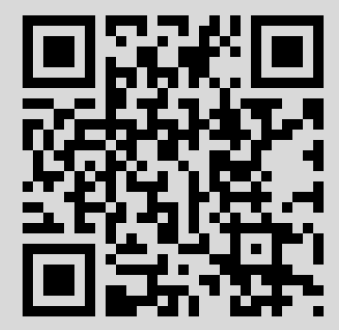




\title{
ОБ ОБЛАСТЯХ ОПРЕДЕЛЕНИЯ $t$-ФУНКЦИЙ
}

\author{
Н. В. Литвинов
}

\begin{abstract}
Частично рекурсивная функция, отличная от пустой или константы, для которой всякая функция сходная с ней изомерна ей, называется $t$-функцией. Доказано, что область определения любой $t$-функции не является ни простым, ни псевдопростым множеством.

Библиография: 3 названия.
\end{abstract}

Через $\mathbb{N}$ обозначим множество всех целых неотрицательных чисел, $A \backslash B$ - разность множеств $A$ и $B$. Если $A \subseteq \mathbb{N}$, то дополнение $\mathbb{N} \backslash A$ обозначим через $\bar{A}$. Под функиией понимаем одноместную частично рекурсивную функцию (ЧРФ). Термин рекурсивная функиия будем применять ко всюду определенным на $\mathbb{N}$ ЧРФ. Обозначать ЧРФ будем строчными греческими буквами $\alpha, \beta, \gamma, \ldots$, а рекурсивные функции - строчными латинскими $f, g, h, \ldots$. Далее $\delta \alpha$ и $\rho \alpha$ обозначают соответственно область определения и область значений функции $\alpha$. Множество $\{x \mid \alpha(x)=a\}$ назовем $a$-уровнем функции $\alpha$ (обозначение $\alpha^{a}$ ). Если $a \in \alpha^{a}$, то $a$ назовем неподвижной точкой функции $\alpha$.

Рекурсивной перестановкой назьвается равнозначная рекурсивная функция, отображающая $\mathbb{N}$ на $\mathbb{N}$.

На множестве всех ЧРФ введем два отношения: сходства и рекурсивного изоморфизма. Говорим, что функция $\alpha$ сходна с функцией $\beta$ (обозначение: $\alpha \sim \beta$ ), если существуют рекурсивные перестановки $f$ и $g$ такие, что $\alpha=f^{-1} \beta g$; функция $\alpha$ рекурсивно изоморфна функции $\beta$ (обозначение $\alpha \equiv \beta$ ), если $\alpha=f^{-1} \beta f$ для некоторой рекурсивной перестановки $f$. Оба введенных отношения являются отношениями эквивалентности. Классы эквивалентных элементов по эквивалентности назовем типами сходства, а по эквивалентности 三- типами рекурсивного изоморфизма. Если две функции рекурсивно изоморфны, то они сходны. Обратное, вообще говоря, не верно. Таким образом, типы сходства состоят из типов рекурсивного изоморфизма.

Роджерсом в [1] была поставлена проблема: какие типы сходства, кроме известных сейчас типов пустой (т.е. нигде не определенной) функции, типа всех функций констант и типа всех универсальных функций, также состоят из единственного типа рекурсивного изоморфизма? Этот вопрос до сих пор остается открытым.

В работе [2] было введено понятие $t$-функции, как функции, отличающейся от пустой функции и всех функций констант, тип сходства которой совпадает с типом рекурсивного изоморфизма. Используя это понятие, проблему Роджерса можно сформулировать так: существуют ли $t$-функции, не являющиеся универсальными? 
В настоящей заметке доказано, что область определения любой $t$-функции не является ни простым, ни псевдопростым множеством.

Приведем результаты, полученные в [2], [3], которые будут использованы в дальнейшем. Если $\alpha-t$-функция, то для нее выполнены следующие свойства.

Свойство Р1 [3]. Область определения функции $\alpha$, а также множество неподвижных точек являются нерекурсивными множествами.

Свойство Р2 [2]. Область значений $\alpha$ есть натуральньй ряд.

Свойство Р3 [2]. Уровни $\alpha$ являются бесконечными попарно рекурсивно изоморфными множествами.

Лемма 1. Пусть $A$ и $B$ - бесконечные рекурсивно перечислимые мнохсества, $A \subseteq B$ и $A=f(B)$ для некоторой рекурсивной перестановки $f$. Тогда числа из множества $B \backslash A$ являются әлементами различных бесконечных ииклов функ$u, u u f$.

ДоказАтЕльство. Так как $f$ - рекурсивная перестановка, то любое натуральное число принадлежит некоторому циклу (конечному или бесконечному) функции $f$. Покажем, что если $x \in B \backslash A$, то $x$ не является элементом никакого конечного цикла $f$.

Пусть $x \in B \backslash A$. Тогда по условию $y=f(x) \in A$. Кроме того, поскольку $f(A) \subseteq A$, то для всех $k$ выполнено $f^{k}(y) \notin B \backslash A$ и, значит, для всех $k f^{k}(y) \neq x$.

Если бы два различных элемента $x$ и $y$ из $B \backslash A$ принадлежали одному бесконечному циклу рекурсивной перестановки $f$, то нашлось бы некоторое $k \geqslant 1$ такое, что либо $f^{k}(x)=y$, либо $f^{k}(y)=x$. Но это невозможно, так как для всякого $k \geqslant 1$ элементы $f^{k}(x)$ и $f^{k}(y)$ попадают во множество $A$. Лемма доказана.

Пусть $\alpha$ - произвольная функция, $A$ - некоторое множество. Для всякого $i=0, \pm 1$, $\pm 2, \ldots$ определим множество $\alpha^{i}(A)$ следуюшим образом:

$$
\alpha^{i}(A)= \begin{cases}\left\{x \mid(\exists y)\left(y \in A \wedge \alpha^{-i}(x)=y\right)\right\}, & \text { если } i<0, \\ \left\{x \mid(\exists y)\left(y \in A \wedge \alpha^{i}(y)=x\right)\right\}, & \text { если } i \geqslant 0,\end{cases}
$$

где $\alpha^{i}(x)=x$, если $i=0$, и $\alpha^{i}(x)=\alpha\left(\alpha^{i-1}(x)\right)$ при $i>0$.

ЛЕмма 2. Пусть $\alpha-t$-функиия, $a$ - неподвижная точка $\alpha$. Тогда найдется рекурсивная перестановка $g$ такая, что

(1) $g(\delta \alpha)=\delta \alpha$

(2) әлементы множества $U=\bigcup_{i=0}^{+\infty} \alpha^{-i}(\{a\})$ принадлежсат различным бесконечным ииклам $g$.

ДоКАЗАТЕЛЬСТВО. Пусть $S_{\alpha}$ - множество неподвижных точек функции $\alpha, b-$ неподвижная точка функции $\alpha$ отличная от $a$. Определим рекурсивную перестановку

$$
f(x)= \begin{cases}b, & \text { если } x=a, \\ a, & \text { если } x=b, \\ x & \text { в остальных случаях }\end{cases}
$$


и положим $\beta=f \alpha$. Через $S_{\beta}$ обозначим множество неподвижных точек функции $\beta$. Очевидно, что $S_{\beta}=S_{\alpha} \backslash\{a, b\}$, а множество $\{a, b\}$ является циклом длины два функции $\beta$. Поскольку $\alpha-t$-функция, то $\alpha=g^{-1} \beta g$ для некоторой рекурсивной перестановки $g$. Введем следующие обозначения:

$$
\begin{gathered}
U_{\alpha}^{k}=\bigcup_{i=0}^{k} \alpha^{-i}\left(S_{\alpha}\right), \quad U_{\beta}^{k}=\bigcup_{i=0}^{k} \beta^{-i}\left(S_{\beta}\right), \quad k \geqslant 0, \\
U_{\alpha}^{\infty}=\bigcup_{i=0}^{+\infty} \alpha^{-i}\left(S_{\alpha}\right), \quad U_{\beta}^{\infty}=\bigcup_{i=0}^{+\infty} \beta^{-i}\left(S_{\beta}\right) .
\end{gathered}
$$

Отметим, что при введенных обозначениях $S_{\alpha}=U_{a}^{0}$ и $S_{\beta}=U_{\beta}^{0}$.

Так как $U_{\beta}^{\infty} \subseteq U_{\alpha}^{\infty}$ и $U \subseteq U_{\alpha}^{\infty} \backslash U_{\beta}^{\infty}$, согласно лемме 1 достаточно показать, что $g\left(U_{\alpha}^{\infty}\right)=U_{\beta}^{\infty}$. Очевидно, что $g\left(U_{\alpha}^{0}\right)=U_{\beta}^{0}$. Допустим теперь, что для некоторого $k \geqslant 2$ выполнено равенство $g\left(U_{\alpha}^{k-1}\right)=U_{\beta}^{k-1}$. Пусть $x \in U_{\alpha}^{k}$. Тогда $\alpha(x)=c$ для некоторого элемента $c$ из $U_{\alpha}^{k-1}$. По индуктивному предположению $g(c) \in U_{\beta}^{k-1}$. Имеем

$$
\beta g(x)=g \alpha(x) \in U_{\beta}^{k-1} \quad \text { и } \quad g(x)=\beta^{-1}(g \alpha(x))=\beta^{-1}(g(c)) \in U_{\beta}^{k} .
$$

Лемма 3. Пусть $\alpha-t$-функиия, $A$ - бесконечное множество. Тогда множество $\alpha^{-1}(A)$ не рекурсивно.

ДокАЗАТЕЛЬСтво. Обозначим через $U$ множество $\alpha^{-1}(A)$.

Случай 1. Множество А рекурсивное кобесконечное. Допустим, что $U$ - рекурсивное множество. Так как $U$ бесконечное, без потери общности можно считать, что $A=U$. Определим рекурсивную перестановку $f$ такую, что $f(A)=\bar{A}$, и положим $\beta=f \alpha$. Очевидно, $\beta(A)=\bar{A}$ и $\beta(\bar{A})=A$ и $\beta$ не имеет неподвижных точек. Противоречие со свойством Р1.

Случай 2. Множество $A$ коконечное. Множество $U$ не может быть рекурсивным, поскольку в этом случае все (кроме, может быть, конечного числа) неподвижные точки $\alpha$ принадлежат $U$. Противоречие со свойством Р1.

Случай 3. Множество $A$ не рекурсивное. Множество $U$ не может быть рекурсивньм, так как в этом случае функция

$$
\chi_{A}(x)= \begin{cases}0, & \text { если }(\exists y)(y \in U \wedge \alpha(y)=x), \\ 1 & \text { в противном случае }\end{cases}
$$

является рекурсивной характеристической функцией множества $A$. Действительно, так как согласно Р 2 область значений $\alpha$ есть натуральный ряд, обязательно найдется $y$ такое, что $\alpha(y)=x$. А так как множество $U$ целиком состоит из уровней $\alpha$, любое такое $y$ принадлежит либо $U$, либо $\bar{U}$.

ТЕорема. Область определения любой псевдопростым множеством. 
ДоКАЗАТЕЛЬСТВО. Пусть $\alpha-t$-функция, $a$ - неподвижная точка функции $\alpha$. Согласно лемме 2 найдется рекурсивная перестановка $g$ такая, что $g(\delta \alpha)=\delta \alpha$ и элементы множества $\bigcup_{i=0}^{\infty} \alpha^{-i}(\{a\})$ принадлежат различным бесконечным циклам $g$. Обозначим через $G$ множество $\{g(x), g g(x), g g g(x), \ldots\}$ (сплинтер рекурсивной перестановки $g$ в точке $g(x))$.

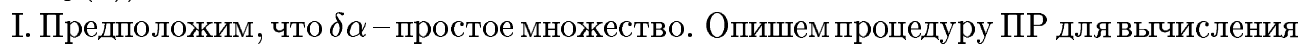
функции $\chi$ в точке $x$. Пусть $s-$ сигнальная переменная, и пусть сначала $s=0$.

ПРОцЕДУРА ПР. Начинаем одновременно вычислять $\alpha(x)$ и перечислять множество $G$. Возможны следующие два случая. (Рассматриваем тот из них, которьй совершится раньше. Если оба случая произойдут одновременно, то для определенности рассматриваем первьй случай.)

Случай 1. Вычислено $\alpha(x)$. Рассмотрим два подслучая.

(a) $\alpha(x)=a$. Полагаем $\chi(x)=1$.

(б) $\alpha(x) \neq a$. Тогда, если $s=1$; полагаем $\chi(x)=0$, иначе $s$ присваиваем значение 1 и вьполняем процедуру ПР с $\alpha(x)$ в качестве $x$.

Случай 2. Вычислено $x$ в $G$. Полагаем $\chi(x)=0$.

Поскольку $g(\delta \alpha)=\delta \alpha$, а $\overline{\delta \alpha}$ - иммунное множество и, следовательно, не может иметь бесконечных РП-подмножеств, то $\overline{\delta \alpha}$ будет целиком состоять из конечных циклов рекурсивной перестановки $g$. Следовательно, всякое натуральное число принадлежит либо некоторому конечному сплинтеру $g$, либо множеству $\delta \alpha$. Учитьвая это, нетрудно видеть, что функция $\chi$ является рекурсивной характеристической функцией множества $\alpha^{-2}(\{a\})$. Но согласно лемме 3 это множество нерекурсивно. Противоречие.

II. Пусть теперь $\delta \alpha-$ псевдопростое множество. Тогда найдется рекурсивно перечислимое множество $B$ такое, что $\delta \alpha \cup B$ простое. В этом случае мы получим процедуру для вычисления рекурсивной характеристической функции множества $\alpha^{-2}(\{a\})$, перечисляя в процедуре ПР множество $G \cup B$ вместо множества $G$.

Теорема доказана.

\section{СПИСОК ЦИТИРОВАННОЙ ЛИТЕРАТУРЫ}

[1] Rogers H. On universal function // Proc. Amer. Math. Soc. 1965. V. 16. № 1. P. 39-44.

[2] Литвинов Н.В. О типах сходства и рекурсивного изоморфизма частично рекурсивных функций // Сиб. матем. ж. 2000. Т. 41. № 1. С. 164-166.

[3] Поляков Е. А. О типах сходства и рекурсивного изоморфизма частично рекурсивных функций // Сиб. матем. ж. 1989. Т. 30. №6. С. 188-192. 